LA--9306-MS

UC-34c

Issued: April 1982

DE82 015080

\title{
The Importance of Elastic Scattering to Particle Direction Determination in Monte Carlo Calculations of DT Reactions in Flight
}

Joseph J. Devaney

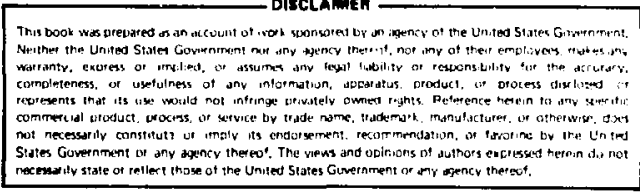


THE IMPORTANCE OF ELASTIC SCATTERING TO PARILCLE DIRECTION DETERMINATION IN MONTE CARLO CALCULATIONS OF DT REACTIONS IN FLIGHT

\author{
by \\ Joseph J. Devaney
}

$\therefore$ BSTRACT

\begin{abstract}
The importance of single, large-angle, nuclearcoulombic, nuclear-hadronic, hadronic-coulombic interference, and multiple nuclear-coulombic scattering is investigated for tritons incident on deuterium, iron, and plutonium for very high temperatures and densities and for ordinary liquid and solid densities at low temperature. Depending on the accuracy desired, we conclude that for $10-\mathrm{keV}$-temperature DT plasmas it is not necessary to include elastic scattering deflection in reaction-in-flight calculations. For higher temperatures or where angular accuracies greater than $10^{\circ}$ are significant or for higher $Z$ targets or for other special circumstances, one must include elastic scattering from coulomb forces.
\end{abstract}

\title{
I. INTRODUCTION
}

In the passage of a deuteron or triton through a DT plasa it is of interest to know the deflection of the incident particle caused by elactic scattering because such deflection will, of course, add to the angular distribution of any subsequent interactions, $1 . e$, to the final extra thernal neutrons. We will show that at low projectile energles the elastic scatering of projectiles by target nuclei is important (Indeed dominant) in the slowing down in high-temperature plasmas and that large angular sattering is possible. however, we belleve that the numbers do not justify the inclusion of elastic scattering in reaction-in-fight (RIF) calculations unless the temperature exceeds $10 \mathrm{keV}$ or so, although we offer a formalisa for the inclusion of the effect if desired. It turns out that nultiple electronic nuclear scatering is the anjor source of the deflections; nuclear forces or hadronic scattering is also inportant. Ten to fifty tines larger deflection occur in heavier ned1a. We give a representative calculation of the various relevant quantitiee. 


\section{THE SCATTERING ANGLE AND CROSS SECTION OF THE COULOMB FIELD DEFLECTION OF A CHARGED PARTICLE; RUTHERFORD SCATTERING}

As a heavy charged particle passes through matter, it loses energy through being deflected by: (a) electrons, (b) nuclei from both coulomb and hadronic elastic scattering plus their interference, (c) nuclear reactions which can change the particle or its parameters, and (d) nuclear bremsstrahlung (a small effect). In the RIF patch, the coulomb interactions with electrons and nuclel are explicitly accounted for in slowing down of the charged particles, but not the resulting angular deviations. The angular deviation of a heavy ion caused by electrons is negligible; that by lons may not be. The possible change in angle in a single collision can be large, although usually with low probability. In this section we g.tve the probability and angles of coulomb deflections, 1.e., deflections by the electrostatic repulsions of ions from the target nuclei. For like particles, particular quantum effects come into play because of the Indistinguishability of the particles that must be represented in the theory. For unlike particles, the famous Rutherford scattering cross section for charged interactions only is

$$
\mathrm{d} \sigma_{R} / \mathrm{d} \Omega=\frac{\mathrm{z}_{1}^{2} \mathrm{z}_{2}^{2} \mathrm{e}^{4}\left(\mathrm{~m}_{1}+\mathrm{m}_{2}\right)^{2}}{4 \mathrm{E}_{0}^{2} \mathrm{~m}_{2}^{2}\left(2 \cdot \mathrm{sin}^{2} \frac{\theta}{2}\right)^{2}}
$$

giving the center-of-mass (CM) differential scattering cross section where 1 refers to the incident particle and 2 to the target. Ze is charge, $m$ is mass, $E_{0}$ is the initial kinetic energy (laboratory system), and $\theta$ is the CM angle of deflection. Equation (1) cannot be used for like particles because particular quantum effects of their distinguishability come into play (see Ref. 1). Note that Eq. (1) blows up at $\theta=0$, that is, $d \sigma_{p} / \alpha=\infty$. Indeed, the total scattering cross section of a "bare" charge is infinite. But bare charges are impossible; they are always neutralized if one goes out far enough, and anyway, the deflections for large cross sections are negligibly small. The Rutherford cross section is indeed strongly peaked forward. The effect is increased in transformation to the laboratory system by the increase of the forward velocity of the center of mass itself. The governing equations ${ }^{2}$ are

$$
\tan \theta_{L}=\sin \theta\left[\left(\frac{m_{1}}{m_{2}}\right)+\cos \theta\right]^{-1}
$$

and

$$
\sigma_{R L}^{\prime}\left(\theta_{L}\right)=\left[1+2\left(\frac{m_{1}}{m_{2}}\right) \cos \theta+\left(\frac{m_{1}}{m_{2}}\right)^{2}\right]^{3 / 2} \cdot\left|1+\left(\frac{m_{1}}{m_{2}}\right) \cos \theta\right|^{-1} \sigma_{R}^{\prime}(\theta)
$$


where the unlabeled variables refer to the $\mathrm{CM}$ system and the variables labeled "L" to the laboratory system.

Computing the monentum given to the incident particle following the collision by watching monenta and energy before and after the collision, and then the postcollision energy of that particle, we can compute the 10s: of energy from the elastic acattering through an angle $\theta_{L}$.

$$
\Delta E=E_{0}\left\{1-\left(\frac{a_{1}}{m_{1}+m_{2}}\right)^{2}\left[\cos \theta_{L} \pm \sqrt{\cos ^{2} \theta_{L}-\left(\frac{m_{1}^{2}-m_{2}^{2}}{m_{1}^{2}}\right)}\right]^{2}\right\}
$$

where the " $+"$ goes with ${ }^{\circ} \mathrm{L} \leq 90^{\circ}$ and the "-" with ${ }^{\theta} \mathrm{L} \geq 90^{\circ}$. Table I givea a numerical ample of these expressions for a 2-MeV triton incident on a deuteron. Unlabeled variables are In $C M$; those labeled " $L$ " are in the laboratory system. Thus with the exception of the aingular point at $\theta_{L}=41.81^{\circ}$, we see that larger angles have much lower cross sections. The singular point at $\left.\theta_{L}=41.8\right)^{*}$ is a polnt of zero measure so $1 t$ does not invalidate the rewark. The singular point is caused by slowdown and the reversal of laboratory angular deflection rate of increase as the $\mathrm{CM}$ velocity exceeds the triton velocity in the $C M$ and occurs for $\cos \theta=m_{2} / m_{1}$ [see Eq. (3)]. For $m_{2}>m_{1}$ these singular points do not occur.

Table I Indeed shows that small-angle scattering is overwhelmingly more likely but does not show the net effect nor compare nuclear scattering with electron scattering. We will compute the mean coulomb scattering angle in Section $V$, but first we calculate the net effect of the various processes by giving the mean rate of energy loss for each.

TALLE I

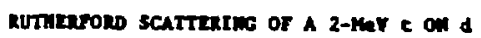

\begin{tabular}{|c|c|c|c|c|c|c|c|c|c|c|}
\hline () & 0 & $10^{\circ}$ & $20^{\circ}$ & $30^{\circ}$ & $60^{\circ}$ & $90^{\circ}$ & $120^{\circ}$ & $131.81^{\circ}$ & $130^{\circ}$ & $180^{\circ}$ \\
\hline${ }^{6} \mathrm{~L}$ & 0 & $2.185^{\circ}$ & $5,60^{\circ}$ & $8.45^{\circ}$ & $16.1^{\circ}$ & $21.8^{*}$ & $23.4^{\circ}$ & $41.81^{\circ}$ & $17.0^{\circ}$ & 0 \\
\hline$\sigma_{R}^{\prime}(b / e r)$ & - & 35.1 & 2.23 & 0.45 & 0.032 & 0.006 & 0.0036 & 0.0029 & 0.0023 & 0.0020 \\
\hline $0_{L L}^{\prime}(b / e r)$ & $\bullet$ & 218.9 & 13.8 & 2.9 & 0.191 & 0.047 & 0.033 & - & 0.0041 & 0.00051 \\
\hline$\Delta E($ MeV) & 0 & 0.0074 & 0.029 & 0.055 & 0.232 & 0.410 & 0.48 & 1.46 & 1.74 & $i .92$ \\
\hline
\end{tabular}


III. RATE OF ENERGY LOSS FROM COULOMB EIASTIC SCATTERING OF A HEAVY ION FROM ELECTRONS AND NUCLEI

To compare the net importance in slowing down by the electrons and the nuclei of a target, we calculate the rates of energy loss and compare. The mean or standard deviation of the net angular deflection is to be found in Section V. Following Longmire, ${ }^{3}$ for electrons labeled 2,

$$
\begin{aligned}
\overline{\mathrm{dE} / \mathrm{dx}}= & -\mathrm{N}_{2} \cdot 4 \sqrt{\pi} \mathrm{z}_{1}^{2} \mathrm{z}_{2}^{2} \mathrm{e}^{4} \cdot(E k T)^{-1 / 2} \cdot \ln \left(2 / \theta_{\mathrm{m}}\right) \cdot \sqrt{\mathrm{m}_{2} / \mathrm{m}_{1}} \\
& \cdot\left[\frac{\mathrm{E}}{(3 / 2) \mathrm{kT}}-1\right],
\end{aligned}
$$

where $N_{2}$ is the electron density, $Z_{2}$ is the electron charge, $E$ is the Incident ion energy, $z_{1} e$ the incident ion charpe, $x$ is the distance, and kT is the temperature of the plasma target. $\theta_{m}$, the minimum CM angle, is the larger of

$$
\text { Classical: } \theta_{m}=z_{1} z_{2} e^{2} / E \lambda_{D}
$$

or

$$
\text { Quantum Mechanical: } \theta_{\mathrm{m}}=\not /\left[\lambda_{\mathrm{D}} \cdot \sqrt{2 \mathrm{mE}}\right] \text {, }
$$

where the Debye length, $\lambda_{D}$, is

$$
\lambda_{D}=\sqrt{k T / 4 \pi N_{e} e^{2}}
$$

Is the reduced mass:

$$
m=m_{1} m_{2} /\left(m_{1}+m_{2}\right)
$$

$N_{e}$ is the electron density.

$$
\begin{aligned}
& \text { For nucle1, } 3 \text { when }\left(m_{2} / m_{1}\right)(E / k T) \gg 1 \text {, we have } \\
& \overline{\mathrm{dE} / \mathrm{dx}}=-\left(\mathrm{N}_{2} / \mathrm{E}\right) \cdot 2 \pi \mathrm{Z}_{1}^{2} \mathrm{Z}_{2}^{2} \mathrm{e}^{4} \cdot \ln \left(2 / \theta_{\mathrm{m}}\right) \cdot\left(\mathrm{m}_{1} / \mathrm{m}_{2}\right),
\end{aligned}
$$

where now $\mathrm{N}_{2}$ is the atov density of target nuclel and $\mathrm{Z}_{2}$ e their charge. 
TANIE II

COULOHBIC ENERG LOSSFS OF A TRITON INCIDENT ON 16-keV TRMPERATURE DEUTTR IUH, IRON, AND PLUTONIUM

\begin{tabular}{|c|c|c|c|c|c|c|}
\hline $\begin{array}{l}\text { Triton } \\
\text { Energy } \\
\text { (MeV) }\end{array}$ & $\begin{array}{c}\text { Energy } \\
\text { Deuterlul } \\
4 \mathrm{~g} / \mathrm{cc}\end{array}$ & $\begin{array}{l}\text { to } \\
\text { at } 16 \mathrm{keV} \text {, } \\
(\mathrm{MeV} / \mathrm{cm})\end{array}$ & $\begin{array}{l}\text { Energy } \\
\text { Iron at } \\
39.3 \text { \& }\end{array}$ & $\begin{array}{l}0.08 \text { to } \\
16 \mathrm{keV}, \\
(\mathrm{MeV} / \mathrm{cm})\end{array}$ & $\begin{array}{r}\text { Fnergy } \\
\text { Plutonlum } \\
200 \mathrm{R} / \mathrm{cc}\end{array}$ & $\begin{array}{l}\text { Lose to } \\
\text { at } 16 \mathrm{keV}, \\
(\mathrm{MeV} / \mathrm{cm})\end{array}$ \\
\hline & $\frac{d E}{d x} \mid$ nuclet & $\frac{d E}{d x} \mid$ electrona & $\frac{d E}{d x} \mid$ nuclet & $\frac{d E}{d x} \mid$ elect rons & $\frac{d x}{d x} \mid$ nuclet & $\frac{d E}{d x} \mid$ electrons \\
\hline 0.1 & 25.2 & 0.58 & 146.5 & 3.4 & 391,8 & B. 3 \\
\hline 1 & 2.9 & 3.6 & 19.3 & 25.8 & 56.0 & 76.8 \\
\hline 2 & 1.5 & 5.6 & 10.3 & 40.4 & 30.5 & 122.5 \\
\hline 5 & 0.62 & 9.7 & 4.5 & 71.6 & 13.5 & 221.5 \\
\hline 10 & 0.32 & 14.6 & 2.4 & 109.4 & 7.3 & 342.5 \\
\hline
\end{tabular}

Choosing rather extreme temperatures and densities of $\mathrm{kT}=16 \mathrm{keV}$, $P_{D}=4 \mathrm{~g} / \mathrm{cm}^{3}$, $P_{\mathrm{Fe}}=39.3 \mathrm{~g} / \mathrm{cm}^{3}$, and $\rho_{\mathrm{Pu}_{\mathrm{u}}}=200 \mathrm{~g} / \mathrm{cm}^{3}$, we get the values of Table II.

Note from Egs. (5) and (1) that the energy loss to free electrons varies as $(\mathrm{kT})^{-3 / 2}$, while the energy loss to nuclet is temperature independent so long as $\left(m_{2} / m_{1}\right)(E / k T) \gg 1$. Therefore, for $E \gg>k$, the doninant loss is to electrons and there is consequently little angular deflection. At high temperatures and incident energies not sufficiently greater than the temperature, nuclear scattering with its possibility of large angles becomes important. Indeed, hadronic and hadronic-coulombic Interference scattering can be Important, as we discuss in the next section.

IV. RATE OF ENERGY LOSS FROM HADRONIC AND HADRONIC-COULOMBIC INTERFERENCE

This section discusses the energy losses from nuclear scattering interactions and their interference with normal electric (coulomblc) elastic scattering. As noted above, because coulombic losses decrease with Increasing temperature, nuclear processes automatically become relatively more important. Nuclear processes are already important at $k T=16 \mathrm{keV}$ for tritons on deuterium as a comparison of Table III with Table II shows.

\section{MULTIPIE COULOMB (OR OTHER) SCATTERING}

Table II shows that at low incident energy and high temperature nuclear-coulonb scattering is an important energy 108s. Yet Table I Inplies that the angle of scattering lo $8 \mathrm{mall}$ but does not say how snall 
the net effect of many scatterings can be. This section gives that result, namely, the angular distribution from multiple coulomb (or any) scattering. Multiple scattering, being a stochastic process, leads to a normal distribution 4,5 in the net or final angle of an incident particle in any plane relative to the original directions. If one chooses orthogonal planes, the two Cartesian normal distributions can then be replaced by polar coordinates and the integration over azimuth performed (the distributions are azimuthally symmetric) to give the radial angle, $\theta$, distribution for finding a particle deflected by $\theta$ if the variance is $\left\langle\theta^{2}\right\rangle$ :

$$
p(\theta) d \theta=\left(2 \theta /\left\langle\theta^{2}\right\rangle\right) e^{-\theta^{2} /\left\langle\theta^{2}\right\rangle} \cdot d \theta
$$

TABLE III

HADRONIC AND HADRONIC-COULOMBIC INTERFERENCE ENERGY LOSS OF A TRITON INCIDENT ON $4 \mathrm{~g} / \mathrm{cm}^{3}$ DEUTERIUM (REF. 1)

$\begin{array}{lcccc}\mathrm{T}_{\mathrm{Tr} i t o n}(\mathrm{MeV}) & 1 & 2 & 5 & 10 \\ \mathrm{dE} / \mathrm{dx}(\mathrm{MeV} / \mathrm{cm}) & \approx 1.6 & 2.19 & 4.76 & 9.21\end{array}$

If the thickness of the target medium is $t$ or $s$ ( $-\rho s$, $\rho$ is density), then define $\theta_{1}$ to be that angle for which there is only one collision with $\theta>\theta_{1}$ in the thickness $t$ or $s$. Then, 4,6

$$
\theta_{1}^{2}=4 \pi N_{2} z_{1}^{2} z_{2}^{2} e^{4} \cdot\left(p^{2} v^{2}\right)^{-1}
$$

where $s$ is in centimeters" $p$ is the relative momentum, $v$ the incident velocity, 1 refers to $C M$ system incident, and 2 to target;

$$
9_{1}^{2}=0.039228 \cdot t \cdot z_{1}^{2} z_{2}^{2}\left(\frac{m_{1}+m_{2}}{m_{2}}\right)^{2} / E_{t}^{2}
$$

for $E_{t}$ in $M e V$ and $t$ in $g / \mathrm{cm}^{2}$; and

$$
\theta_{1}^{2}=0.039228 \cdot \frac{\rho_{2}}{A_{2}} \cdot z_{1}^{2} z_{2}^{2}\left(\frac{m_{1}+m_{2}}{m_{2}}\right)^{2} / E_{t}^{2}
$$

CM system 
where thickness $s$ is in centimeters, density $\rho_{2}$ in $\mathrm{g} / \mathrm{cm}^{3}$, triton energy $E_{t}$ in $\mathrm{MeV}$, and $\mathrm{A}_{2}$ is the atomic weight of the target?

The angular standard deviation, $\sigma$, squared of the distribution $1 \mathrm{~s}$ gIven by: 4,6

$$
\sigma^{2} \equiv\left\langle\theta^{2}\right\rangle=2 \theta_{1}^{2} \ln \left(\theta_{1} / \theta_{m}\right) \text {. CM system }
$$

$\theta_{m}$ is the mintmum angle of scattering, given for plasmas in Eqs. (6) and $(7)$.

A more physically meaningful measure of a distribution is the median (the 50th percentile) or for errors, the probable error, which we labe $1 \tau$ and define

$$
0.5=\int_{0}^{\tau} p(\theta) d \theta .
$$

Substituting Eq. (11) and performing the integration give

$$
\tau=\sqrt{\ln 2} \cdot \sqrt{\left\langle\theta^{2}\right\rangle}=0.832550 .
$$

The transformation [Eq. (2)] from the CM to the laboratory system is, for $\mathrm{CM}$ angles not too large, $\mathrm{i} . \mathrm{e}, \cos \theta<\left(\mathrm{m}_{\text {incident }} / \mathrm{m}_{\text {target }}\right)$, an iso-
morphism and the median transforms into the median.

Choosing a high temperature, $\mathrm{kT}=16 \mathrm{keV}$, and very dense materials we give in Table IV the median, $T$, of the multiple coulomb scattering angular distributions for tritons incident on deutertum, 1 ron, and plutoniun calculated from Eqs. (14), (15), (17), and (2) for a distance $s=0.01 \mathrm{~cm}$ and a flxed incident energy. Note that at lower incident energies and at higher charges of the target atoms, the energy $108 \mathrm{~s}$ is appreciable in passing through the foll so that the assunption of a fixed energy of the projectile is Inaccurate and the numbers should be used for comparison only. Such comparative numbers are marked with an asterisk (*).

It is evident from Table IV, with an eye to the range of the triton from $\mathrm{dE} / \mathrm{dx} /$ nuclej $+\mathrm{dE} / \mathrm{dx} /$ electrons in Table II, that the coulombic multiple scátering leads Eocterons net scatterings of 6 to $10^{\circ}$ over the range of the triton to just below about $1 \mathrm{MeV}$. In heavier material the multiple smal1-angle electronfc scattering is much larger. At lower temperatures the median scattering decreases weakly as 
MULTIPLE COULOMB SCATTERING THROUGH $0.01 \mathrm{~cm}$ THICKNESS** TEMPERATURE, $\mathrm{kT}=16 \mathrm{keV}$

Target

Isotope

and mass density
Incident Triton Energy

$E_{t}(\mathrm{MeV})$
Minimum Angle of Scatter

$\theta_{m}$ (radians)
Laboratory Scattering Angle Median

$\tau_{\mathrm{L}}$ (degrees)
$\left(4 \mathrm{~g} / \mathrm{cm}^{3}\right)$
0.1
4. $18(-5)$
$39.7^{\circ}$
1.0
7. $65(-6)$
$5.7^{\circ}$
2
$5.41(-6)$
$2.8^{\circ}$
5
3. $42(-6)$
$1.1^{\circ}$
10
2. $42(-6)$
$0.53^{\circ}$
$\left(39.3^{\mathrm{Fe}} \mathrm{g} / \mathrm{cm}^{3}\right)$
0.1
1. $39(-3)$
$>180^{\circ} *$
1.0
1. $39(-4)$
$84.3^{\circ} *$
2
$6.96(-5)$
$41.6^{\circ} *$
5
$2.79(-5)$
$16.6^{\circ}$ *
10
$1.39(-5)$
$8.3^{\circ} \star$
$\left(200 \mathrm{Pu} / \mathrm{cm}^{3}\right)$
0.1
$9.2(-3)$
$>180^{\circ} *$
1. 0
$9.2(-4)$
$>180^{\circ} *$
2
$4.6(-4)$
$159.4^{\circ}$
5
1. $84(-4)$
$63.2^{\circ}$ *
10
$9.2(-5)$
$31.6^{\circ}$ *

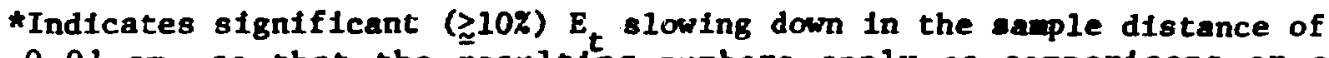
$0.01 \mathrm{~cm}$, so that the resulting numbers apply as comparisons or at $E_{t}$ only.

* *Computed on the basis that the $\mathrm{kT}=16 \mathrm{keV}$ motion of the target 18 negliglble, which 18 certalnly not a good approxination for $E_{t}=$ $100 \mathrm{keV}$. 
(1/2) lnkT [Eqs. (8), (6), (7), and (15)]. Indeed, for comparison

we include the laboratory median, $\tau$, for the scattering of tritons

by $0.01 \mathrm{~cm}$ of deuterium, iron, and plutonium at solid or liquid

densities and at nonplasma temperatures. See Table V.

For the minimum scattering angle in un-ionized media we used the larger of : ${ }^{6}$

$$
\text { Classical: } \theta_{\mathrm{m}}=3.8 \mathrm{z}_{2}^{4 / 3} \mathrm{e}^{2}\left(\mathrm{~m}_{1}+\mathrm{m}_{2}\right) /\left(2 \mathrm{~m}_{2} \mathrm{E}_{\mathrm{t}} \mathrm{a}_{0}\right)
$$

or

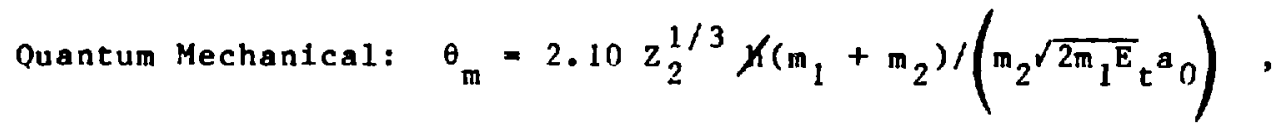

where $a_{0} \equiv \not h^{2} / \mathrm{me}_{\mathrm{e}} \mathrm{e}^{2}$ is the Bohr radius, 1 zefers to the incident triton, and 2 to the target nucleus. The values used are found in Table $v$.

TABLE $V$

MULTIPLE COULOMB SCATTERING

\begin{tabular}{|c|c|c|c|c|}
\hline $\begin{array}{l}\text { Target Isotope } \\
\text { and Mass Density }\end{array}$ & $\begin{array}{l}\text { Inc1dent } \\
\text { Triton } \\
\text { Energy (MeV) }\end{array}$ & $\begin{array}{c}\text { Minfmum Scattertig } \\
\text { Angle } \\
\theta_{\text {(radians) }}\end{array}$ & $\begin{array}{l}\text { Rate of Energy } \\
\text { Loss With Dotance } \\
\mathrm{dE}_{\mathrm{t}} / \mathrm{dx}(\mathrm{MeV} / \mathrm{cm})\end{array}$ & $\begin{array}{l}\text { Median Laboratory } \\
\text { Scattering angle } \\
T_{L} \text { (degrees) }\end{array}$ \\
\hline \multirow{5}{*}{$\left(0.14^{\mathrm{g}} / \mathrm{cs}^{3}\right)$} & 0.1 & $1.29(-3)$ & $2.5(2)$ & $4.82^{\circ}$ \\
\hline & 1.0 & $2.61(-4)$ & $1.2(2)$ & $0.44^{\circ}$ \\
\hline & 2 & $1.84(-4)$ & 67 & $0.21^{\circ}$ \\
\hline & 5 & $1.17(-4)$ & 33 & $0.077^{\circ}$ \\
\hline & 10 & $8.25(-5)$ & 17 & $0.036^{\circ}$ \\
\hline \multirow{5}{*}{$\begin{array}{c}\mathrm{Fe} \\
\left(7.96 \mathrm{~g} / \mathrm{cm}^{3}\right)\end{array}$} & 0.1 & 0.042 & $1.6(3)$ & $\circ>180^{\circ}$ \\
\hline & 1.0 & 0.0042 & $1.8(3)$ & $25.7^{\circ}$ \\
\hline & 2 & $0 . n 021$ & $1.3(3)$ & $12 . B^{\circ}$ \\
\hline & 5 & 0.00084 & $8.0(2)$ & $5.1^{\circ}$ \\
\hline & 10 & 0.00042 & $4.9(2)$ & $2.6^{\circ}$ \\
\hline \multirow{5}{*}{$\left(19.84 \mathrm{gu} / \mathrm{cm}^{3}\right)$} & 0.1 & 0.224 & $1.9(3)$ & $0>180^{\circ}$ \\
\hline & 1.0 & 0.0224 & $2.2(3)$ & $64.8^{\circ}$ \\
\hline & 2 & 0.0119 & $1.5(3)$ & $32.3^{\circ}$ \\
\hline & 5 & 0.00448 & $9.3(2)$ & $12.9^{\circ}$ \\
\hline & 10 & 0.00226 & $6.4(2)$ & $6.5^{\circ}$ \\
\hline
\end{tabular}

LIOUID AND SOLID DENSITIES (UN-IONIZED), $0.01-\mathrm{cm}$ THICKNESS

*The adian values in this colum are computed on the basis that the energy remains as given in colum two throughout the thickness of $0.01 \mathrm{~cm}$, which colum four shows cannot be true. Hence, these values are so to spas initial values per $0.01-\mathrm{cm}$ thickines and are meant to be comparative only. 
$d E_{t} / d x$ is taken from the encyclopedic work of Anderson and Ziegler. 7 For the rest of Table $V$ we used Eqs. (14), (15), (17), and (2).

\section{DISCUSSION AND CONCIUSIONS}

At very high temperatures, because the eqgrgy loss of a charged particle to electrons varles roughly as (kT) $3 / 2$ [Eq. (5)], the usual dominant electron losses (Table $V$ ) begin to be dominated by nuclear losses at 16-keV temperature (Table II), especially at lower incident energy. Nuclear interactions imply much greater angular deflections, although the greatest probability still is for slight deflections

(Table I). hiwever, two questions remain: (1) can the multiple nuclear coulomb scatterings add up to a significant net deflection, and (2) what is the effect of hadronic (i.e., nuclear forces) scattering? If $10^{\circ}$ (or $20^{\circ}$ ) or so is a negligible deflection, one can ignore multiple coulomb elastic scattering (Table IV) of triton on deuterons or deuterons on tritons at $\mathrm{kT} \cong 16 \mathrm{keV}$, but not on higher $\mathrm{Z}$ targets. For higher $\mathrm{Z}$ or higher temperatures or for better than $10-20^{\circ}$ angular accuracy over the full range of a deuteron or triton, one is going to have to sample a normal distribution [Eq. (11), two dimensional] over the deflection angle with variance given by Eq. (15). In practice, one suspects that each case might have to be examined individually to see if this complication is really necessary.

Comparing Tables III and II for tritons on deuterium we see that already at $16-\mathrm{keV}$ temperature the hadronic energy loss is significant. And, as a rule of thumb, such scatterings at low incident energies always involve large CM angular deflections. The large motion of the $\mathrm{CM}$ in deuterium on triton or triton on deuter um ameliorates the large deflections to a degree.

At lower temperatures (the extremes of low temperatures where the targets are not ionized are given in Table $V$ ), the rate of energy loss to the electrons is so great that the chance of large-angle scattering by the nuclei is reduced to negligible. Note that the large angles shown in Table $V$ are often not realizable because energy degradation is so swift that the triton range is less than the thickness, $0.01 \mathrm{~cm}$, for which the net deflections were calculated.

We conclude (depending on the accuracy desired) that for, say, $\cong 10 \mathrm{keV}$ DT plasma temperatures it is not necessary to include elastic scatte deflections in the RIF patch. For higher DT temperatures or where $10^{\circ}$ is an important random deflection or for special circumstances, i.e., where rare large scattering could be important, elastic scattering from nuclear forces and multiple elastic scattering from coulomb forc. must be included.

The angular deflections from multiple sc ttering of heavy ions from electrons are taken to be negligible. 


\section{ACKNOWLEDGEMENTS}

It is a pleasure to acknowledge extreme parameters furnished by M. I. Shepard and R. L. Osborne.

\section{REFERENCES}

1. J. J. Devaney and M. L. Stein, "Plasma Energy Deposition from Nuclear Elastic Scattering," Nucl. Sci. Eng. 46, 323 (1971).

2. P. I. Richards, Manual of Mathematical Physics (Pergamon Press, Inc., 1959), P. 146.

3. C. L. Longmire, Elementary Plasma Physics (John Wiley and Sons, Inc., New York, 1963).

4. H. A. Bethe and J. Ashkin, in Experimental Nuclear Physics, E. Segrè, Ed. (John Wiley and Sons, Inc., New York, 1953), pp. 283, 285.

5. C. Eisenhard and M. Zelen in Handbook of Physics, 2nd ed., E. U. Condon and H. Odishaw, Eds. (McGraw-Hill Book Co., New York, 1967), Pp. 1-178.

6. N. F. Mott and H. S. W. Massey, The Theory of Atomic Collisions, 3rd ed. (Clarendon Press, Oxford, 1965), P. 629.

7. H. H. Andersen and J. F. Ziegler, Hydrogen Stopping Powers and Ranges in all Elements (Pergamon Press, Inc., New York, 1977). 